Piezoelectric MEMS generators fabricated with an aerosol deposition PZT thin film

This article has been downloaded from IOPscience. Please scroll down to see the full text article.

2009 J. Micromech. Microeng. 19065014

(http://iopscience.iop.org/0960-1317/19/6/065014)

The Table of Contents and more related content is available

Download details:

IP Address: 140.112.39.51

The article was downloaded on 28/12/2009 at 04:26

Please note that terms and conditions apply. 


\title{
Piezoelectric MEMS generators fabricated with an aerosol deposition PZT thin film
}

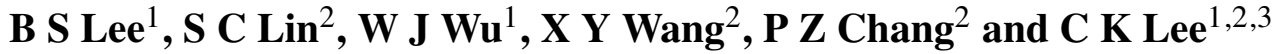 \\ ${ }^{1}$ Engineering Science \& Ocean Engineering, National Taiwan University, Taipei, Taiwan \\ 2 Institute of Applied Mechanics, National Taiwan University, Taipei, Taiwan \\ ${ }^{3}$ Industrial Technology Research Institute (ITRI), Hsinchu, Taiwan \\ E-mail: wjwu@ntu.edu.tw and cklee@ntu.edu.tw
}

Received 30 January 2009, in final form 5 April 2009

Published 20 May 2009

Online at stacks.iop.org/JMM/19/065014

\begin{abstract}
In this paper, we present the development of two piezoelectric MEMS generators, $\{3-1\}$ mode and $\{3-3\}$ mode, which have the ability to scavenge mechanical energy of ambient vibrations and transform it into useful electrical power. These two piezoelectric MEMS generators are of cantilever type made by a silicon process and which can transform mechanical energy into electrical energy through its piezoelectric PZT layers. We developed a PZT deposition machine which uses an aerosol deposition method to fabricate the high-quality PZT thin film efficiently. Our experimental results show that our $\{3-1\}$ mode device possesses a maximum open circuit output voltage of $2.675 \mathrm{~V}_{\mathrm{P}-\mathrm{P}}$ and a maximum output power of $2.765 \mu \mathrm{W}$ with $1.792 \mathrm{~V}_{\mathrm{P}-\mathrm{P}}$ output voltage excited at a resonant frequency of $255.9 \mathrm{~Hz}$ under a $2.5 \mathrm{~g}$ acceleration level. The $\{3-3\}$ mode device possessed a maximum open circuit output voltage of $4.127 \mathrm{~V}_{\text {P-P }}$ and a maximum output power of $1.288 \mu \mathrm{W}$ with $2.292 \mathrm{~V}_{\text {P-P }}$ output voltage at its resonant frequency of $214 \mathrm{~Hz}$ at a $2 \mathrm{~g}$ acceleration. We also compared the output characteristics of both the $\{3-1\}$ mode and the $\{3-3\}$ mode piezoelectric MEMS generators which were both excited at a $2 g$ acceleration level.
\end{abstract}

(Some figures in this article are in colour only in the electronic version)

\section{Introduction}

Over the past few years, the development of wireless sensor network applications has generated much interest. Research on the various ways to power wireless sensor devices has become more and more important [1-3]. Unlike portable devices such as cell phones and PDAs where the batteries can be recharged or replaced regularly, these days, most micro sensors are powered by embedded batteries. Therefore, the life of a battery is a major constraint when trying to extend the convenience of micro sensors. With the advent of lowpower electronic designs and improvements in fabrication, technology has progressed toward the possibility of selfpowered sensor nodes and micro sensors [4].

Much research has been focused on harvesting electric power from various ambient energy sources, including solar power, thermal gradients and vibrations [5]. Among these energy sources, solar power was the first to be proposed as an alternative power source. However, this mature solar power technology is only applicable in an environment where there is enough illumination requiring a large enough area of solar panel. When comparing all possible energy sources, mechanical vibration is a potential power source that is easily accessible through micro-electromechanical systems (MEMS) technology [6, 7]. Mechanical vibration energy can be converted into usable electrical energy through piezoelectric, electromagnetic and electrostatic transducers. The piezoelectric transducer is considered a potential choice when compared with electromagnetic and electrostatic transducers due to its high energy density [8]. There were already many successful vibration harvesting devices reported $[7,9,10]$, which were mainly based on different piezoelectric structure designs and different optimal interface circuits. Piezoelectric material that has been found to have the ability to 
convert vibration energy to electric power has sparked much attention as it was attractive for use in MEMS applications $[9,11-13]$.

Shen et al [13] and Fang et al [11] successfully developed a PZT MEMS power-generating device based on the $\{3-1\}$ mode of piezoelectric transducers that uses top and bottom laminated electrodes. Marzencki et al [14] developed a MEMS generator based on the $\{3-1\}$ mode but using AlN as a piezoelectric material. The device was demonstrated to integrate with an ASIC power management circuit in an SoP (system on a package). Jeon et al [9] developed a PZT MEMS power-generating device based on the $\{3-3\}$ mode of piezoelectric transducers using top-side interdigitated electrodes. Although different piezoelectric modes were adopted for the piezoelectric MEMS power-generating devices mentioned above, the structure of the devices has all been identical in that they have all been of a cantilever type with a single layer PZT fabricated using a sol-gel process supported by a silicon-based beam structure. As a result of the different configurations of the piezoelectric MEMS power-generating devices based on $\{3-1\}$ and $\{3-3\}$ piezoelectric properties, the characteristics of the power output and generated voltage with respect to the optimal resistive load obtained have been different.

In this paper, we present the development of both the $\{3-1\}$ and the $\{3-3\}$ mode piezoelectric MEMS generators. These two piezoelectric MEMS generators are of a cantilever type made using a silicon process which transforms energy by way of the piezoelectric PZT layer. The $\{3-1\}$ mode piezoelectric MEMS generator was laminated with a PZT layer sandwiched between electrodes while the $\{3-3\}$ mode was composed of interdigitated electrodes at the top. Unlike the PZT sol-gel process that is suitable for fabricating thin film with thicknesses of 1-2 $\mu \mathrm{m}$, our own developed PZT deposition machine adopts a 'jet-printing' approach which is based on an aerosol deposition method and was developed and constructed in order to fabricate a high-quality PZT thin film more efficiently.

For fabricating the piezoelectric MEMS generators, a beam structure was manufactured and then covered with a PZT layer with a laminated top and bottom electrode or with interdigitated electrodes. A proof mass was built at the tip of the beam to adjust the structure resonant frequency of the piezoelectric MEMS generator to fit the most adaptable frequency to match the ambient vibration of the surroundings. To harvest the vibration energy from the surroundings, the beam structure was designed to operate at resonant frequency for maximum stress and strain so as also to maximize the electric power output. The output performance and characteristics for both the $\{3-1\}$ mode and the $\{3-3\}$ mode piezoelectric MEMS generators were evaluated using the same dimensions and with the same materials, with the exception of the differing electrode configuration and dimensions of the proof mass. The two devices were then compared for resonance frequency, output power, output voltage and optimal resistive load.

\section{Design concepts}

For self-powered sensor node applications and micro sensor applications, a MEMS generator can be designed to operate around the environment vibration frequency and to convert mechanical vibration energy into electrical power. In order to obtain the maximum power output, the environmental conditions that include vibration frequency and acceleration level for harvesting the vibration energy should be known. For the purpose of converting vibration energy into electric power, a cantilever beam structure with PZT material was adopted. In both the $\{3-1\}$ mode device where the stress was perpendicular to the electric field and in the $\{3-3\}$ mode device where the stress was parallel to the electric field, piezoelectric material was used in the cantilever beam structure [15]. Figure 1 shows the configurations of the piezoelectric MEMS generators. The piezoelectric MEMS generator consisted of a proof mass and a silicon micro-cantilever beam on which a piezoelectric PZT layer was deposited. The $\{3-1\}$ mode piezoelectric MEMS generators were composed of upper and lower electrodes on the PZT materials. On the other hand, the $\{3-3\}$ mode piezoelectric MEMS generators were made up of interdigitated electrodes on top of the PZT material. For the $\{3-1\}$ mode piezoelectric MEMS generator, the generated open circuit voltage $(V)$ can be evaluated by [9]:

$$
V=\sigma_{x x} g_{31} H
$$

where, $\sigma_{x x}$ is the stress in the $x$-direction, $H$ is the thickness of the piezoelectric material and $g_{31}$ is the piezoelectric coefficient. When the micro-cantilever was subjected to ambient vibrations, mechanical stress was induced inside the PZT layer due to a sufficient inertial force provided by the proof mass. At such time, the PZT layer converted the mechanical strain energy into electrical energy and the generated charges were extracted through the electrodes. Since the output was an ac signal, it was able to pass the rectifier to obtain a dc voltage for use as a power source which can be used for a load or a battery. From equation (1), we can see that the thicker the PZT layer, the higher the generated voltage of the device will be. Therefore, the thickness of the PZT layer should be thick enough to generate enough output voltage. For the $\{3-3\}$ mode piezoelectric MEMS generator, due to the interdigited electrode configuration, the piezoelectric material was poled alternatively along the $+x$ and $-x$ directions. The generated open circuit voltage $(V)$ can then be evaluated by [9]:

$$
V=\sigma_{x x} g_{33} L,
$$

where $L$ is the gap between the interdigitated electrodes. From equation (2), we can see that the output voltage will be proportional to the gap of the interdigitated electrodes. Therefore, the output voltage can be adjusted by the fabrication process easily to meet the requirement of passing through the rectifier. In fact, the poling direction of the $\{3-3\}$ mode piezoelectric MEMS generator was not a straight line along the $x$-direction. The PZT material poled by the interdigitated electrode results in a non-uniform poling direction [15]. The material under the electrodes is typically not used because it does not pole correctly. The deeper the distance from the surface of the PZT material, the less effective the poling 


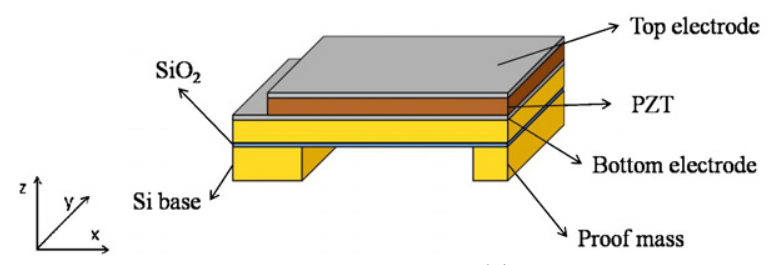

(a)

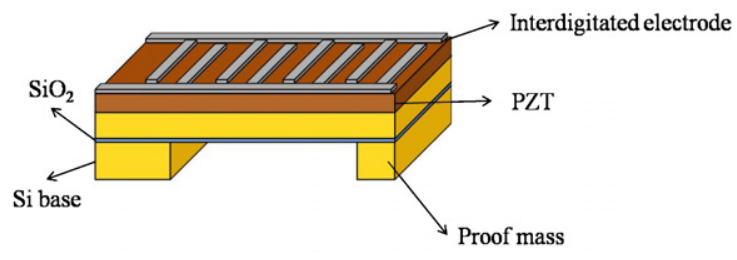

(b)

Figure 1. Schematic diagram of the piezoelectric MEMS generators: (a) $\{3-1\}$ mode configuration, $(b)\{3-3\}$ mode configuration.

electric field strength will be. This causes the efficiency drop for the $\{3-3\}$ mode piezoelectric MEMS generator when compared to the $\{3-1\}$ mode piezoelectric MEMS generator.

The two modes of piezoelectric MEMS generators in this paper were designed to incorporate a $3000 \times 1500 \mu \mathrm{m}^{2}$ size cantilever beam structure with a $11 \mu \mathrm{m}$ thickness comprised of a $5 \mu \mathrm{m}$ piezoelectric PZT layer and a $1 \mu \mathrm{m} \mathrm{SiO}{ }_{2}$ at the bottom of the beam structure. For the $\{3-3\}$ mode piezoelectric MEMS generator, the interdigitated electrodes were fabricated with $30 \mu \mathrm{m}$ widths and $30 \mu \mathrm{m}$ gaps. The proof masse for the two piezoelectric MEMS generators were fabricated under the beam structure with dimensions of $500 \times 1500 \times 500 \mu \mathrm{m}^{3}$ for the $\{3-1\}$ mode and $750 \times 1500 \times 500 \mu \mathrm{m}^{3}$ for the $\{3-3\}$ mode. A different proof mass dimension was used in the two mode devices to demonstrate the ability of the structure to adjust the resonant frequency.

\section{PZT fabrication method}

We fabricated the PZT layer using an aerosol deposition method which has been proven to be a quick, efficient and easyto-pattern MEMS process [16, 17]. The aerosol deposition equipment deposited the PZT film up to $0.1 \mu \mathrm{m} \mathrm{min}^{-1}$. Figure 2 shows the schematic diagram of the aerosol deposition equipment. The PZT powder with a particle size smaller than $1 \mu \mathrm{m}$ in diameter was put in a continuously vibrating powder chamber in order to suspend the PZT particles. Nitrogen or helium gas was connected to the powder chamber with a gas flow rate of 4-6 $1 \mathrm{~min}^{-1}$ so as to bring the PZT particles through the nozzle and into the deposition chamber. With the deposition chamber in a vacuum, the pressure difference between the power chamber and the deposition chamber accelerated the PZT particles and forced them to jet out from the nozzle inside the deposition chamber and deposit onto the wafer surface with high speed. The wafer substrate was then carried by an $\mathrm{X}-\mathrm{Y}$ moving stage so that deposition over the entire area of the PZT took place. Both the flow rate of the inlet gas and the scan speed of the $\mathrm{X}-\mathrm{Y}$ moving stage were then used to control the deposition rate and the roughness of the deposited PZT layer. Figure 3(a) shows the SEM photography of the PZT layer as deposited by aerosol deposition with a thickness of up to $28 \mu \mathrm{m}$.

A lift-off method was adopted to pattern the PZT layer that was deposited by the aerosol deposition machine. A photoresist with suitable hardness and adhesion between the photoresist and PZT powder was needed for the lift-off process to prevent damage to the photoresist during processing and to

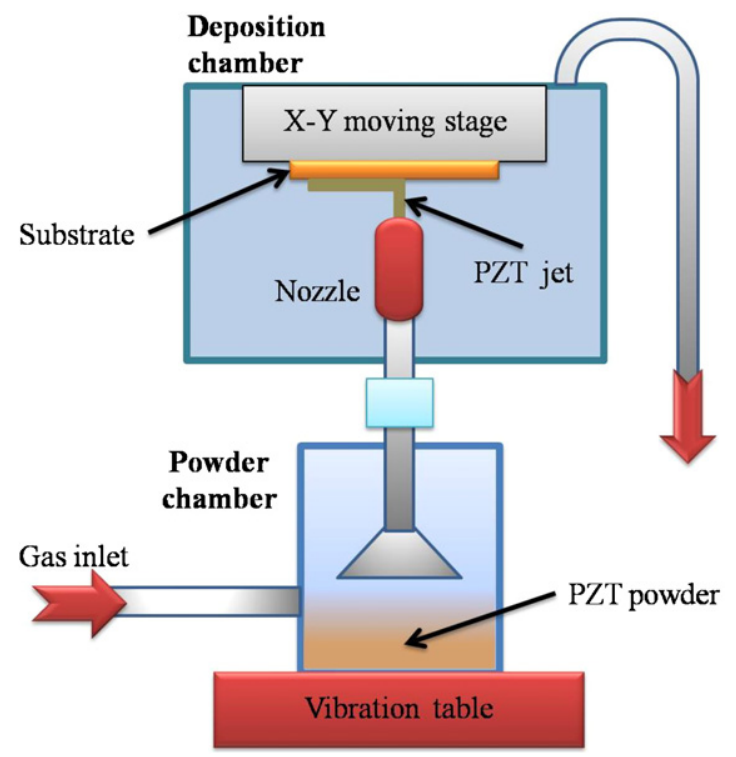

Figure 2. Schematic diagram of the aerosol deposition machine.

limit accumulation of the PZT powder at the sidewall. A photoresist KMPR-1050 (MicroChem Corp.) or THB-151 N (JSR Micro Inc.) was used in this work. Figure 3(b) shows the SEM photograph of the sidewall of the PZT layer patterned by the lift-off method.

Although the PZT film was deposited at room temperature, an annealing process was still required to improve the characteristics of the material. To investigate the effects at different annealing temperatures, the relationship between polarization and the electric field of the annealed PZT film with $5 \mu \mathrm{m}$ in thickness at different annealing temperatures was undertaken using a ferroelectric analyzer (TF ANALYZER 2000). Figure 4 shows the measured P-E hysteresis curves. The applied electrical field was $75 \mathrm{MV} \mathrm{m}^{-1}$ at $100 \mathrm{~Hz}$. The remnant polarizations were $7-9.3 \mu \mathrm{C} \mathrm{cm}^{-2}$ after annealing above $450{ }^{\circ} \mathrm{C}$, which shows much improvement when compared to non-annealed PZT layers. Our experimental results show that the coercive field decreased with respect to an increase in annealing temperature.

The crystalline phase of the deposited PZT layer associated with the different annealed temperatures can be characterized by $\mathrm{x}$-ray diffraction (XRD). The nonannealed crystalline phase was used as a reference point (see figure 5). Our findings indicate that a perovskite phase in the PZT powder remains after a $650{ }^{\circ} \mathrm{C}$ annealing process. In our study, the annealing temperature was set at $650{ }^{\circ} \mathrm{C}$. Therefore, after the PZT film was deposited, it was then 


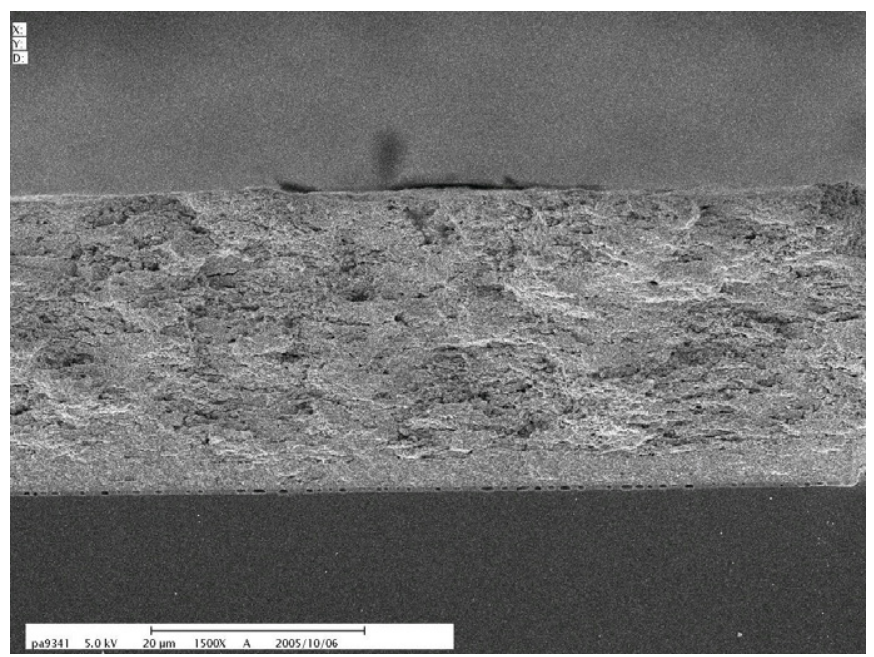

(a)

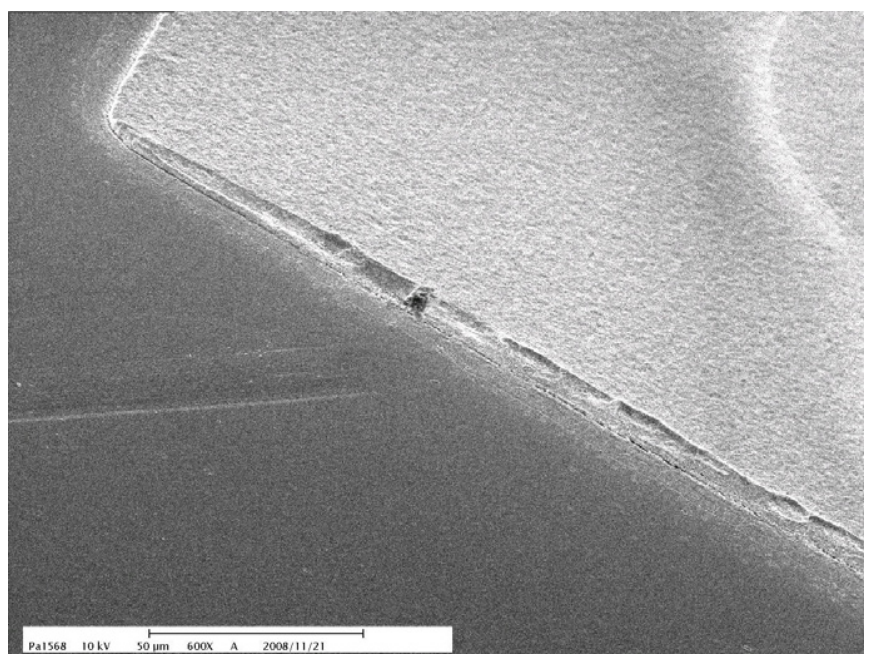

(b)

Figure 3. SEM photograph of the PZT layer after deposition: $(a)$ cross-sectional view of $28 \mu \mathrm{m}$ thickness, and $(b)$ patterned PZT layer by the lift-off method.

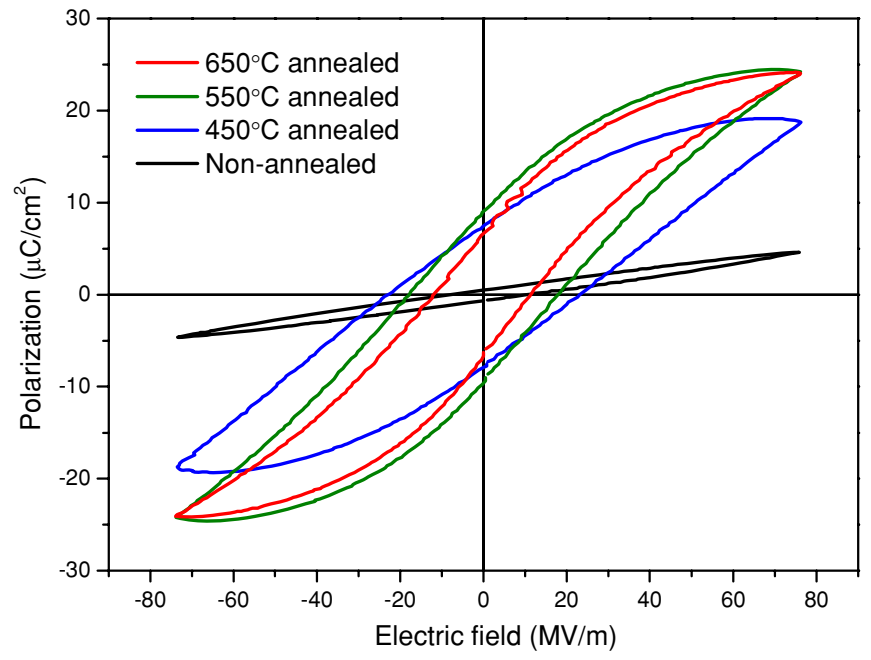

Figure 4. P-E hysteresis curve of a $5 \mu \mathrm{m}$ PZT layer at different annealing temperatures.

annealed at $650{ }^{\circ} \mathrm{C}$ for $3 \mathrm{~h}$ in a furnace and then cooled to room temperature. It should be noted that PZT microstructures will crack easily when the annealing temperature is higher than $700{ }^{\circ} \mathrm{C}$. Similarly, acceptable piezoelectric constants cannot be obtained for annealing temperature lower than $450{ }^{\circ} \mathrm{C}$ [17].

\section{Fabrication process}

The piezoelectric MEMS generator was a laminated cantilever structure which was composed of a supporting silicon membrane, a piezoelectric layer, and laminated electrodes or interdigitated electrodes. The proof mass, made of silicon, was built at the free end of the cantilever beam for tuning the resonant frequency. Most of the process steps were undertaken in a standard clean room environment. The piezoelectric material PZT thin film deposition was deposited using our own developed aerosol deposition machine. The fabrication

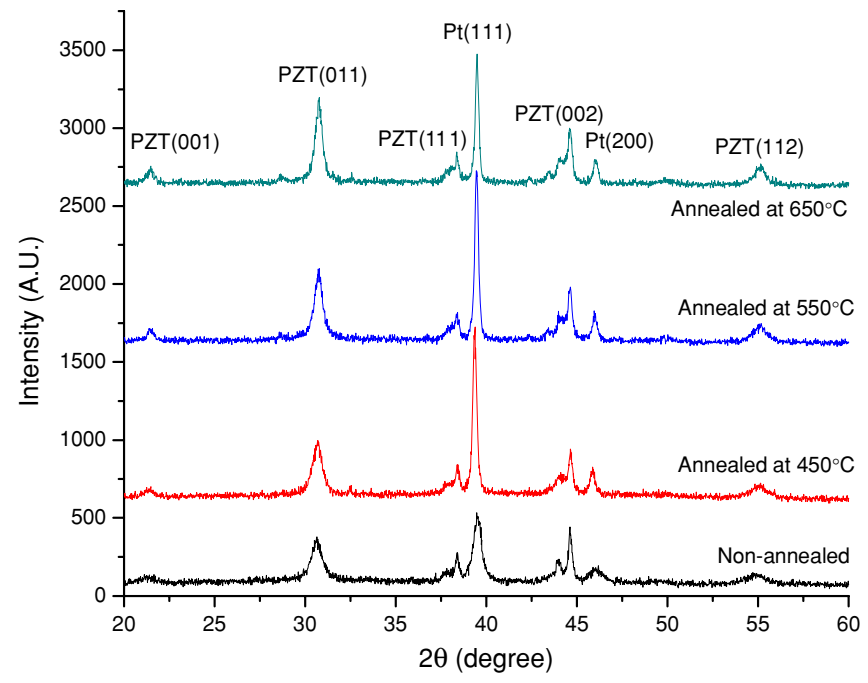

Figure 5. Results of the XRD scan of the PZT layers at different annealing temperatures.

process procedure for the $\{3-1\}$ and $\{3-3\}$ configurations were as follows.

\subsection{The $\{3-1\}$ mode piezoelectric MEMS generator}

Figure 6 shows the fabrication process of the $\{3-1\}$ mode piezoelectric MEMS generator. The SOI wafer with a $5 \mu \mathrm{m}$ device layer and a $1 \mu \mathrm{m}$ buried oxide layer was used in the process. First, the bottom electrode was deposited with a $30 \mathrm{~nm} \mathrm{Ti}$ and $220 \mathrm{~nm}$ Pt on the top side of the SOI wafer using an e-beam evaporator and then a lift-off process was adopted for patterning the electrode shapes. A $5 \mu \mathrm{m}$ PZT layer was then deposited onto the bottom electrode using the aerosol deposition method described above followed by patterned with the lift-off method using a THB-151 N photoresist and then annealed at $650{ }^{\circ} \mathrm{C}$ for $3 \mathrm{~h}$. An e-beam evaporator was then used to deposit the top electrode with $30 \mathrm{~nm}$ Ti and $220 \mathrm{~nm} \mathrm{Pt}$ and then patterned. The beam shape was defined and etched on 
(a)

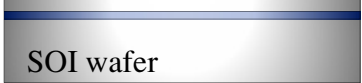

(b)

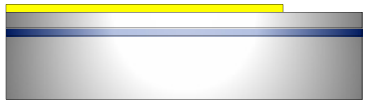

(c)

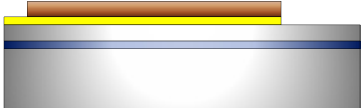

(d)

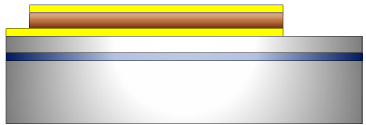

(e)

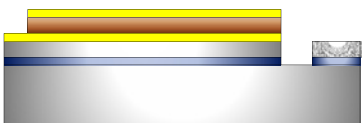

(f)

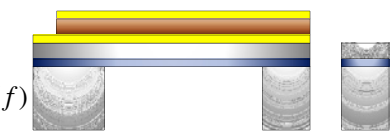

$\mathrm{Si}$

$\mathrm{SiO}_{2}$

$\mathrm{Ti} / \mathrm{Pt}$

PZT

Figure 6. Processing procedure flowchart for $\{3-1\}$ mode piezoelectric MEMS generator: (a) SOI wafer preparation, (b) bottom electrode deposition and patterning, (c) PZT deposition and patterning, $(d)$ top electrode deposition and patterning, $(e)$ beam shape etching and $(f)$ back-side etching and released beam.

the top-side DRIE, and the buried oxide layer was etched out using RIE at the same time. Finally a DRIE process was then used to etch the wafer from the back side until the beam was released. The proof mass was made at the same time and its size was adjusted during the etching to the back side. The PZT layer was then poled under a high electric field. The device was heated up to $160{ }^{\circ} \mathrm{C}$ using a hot plate, followed by poling under $100 \mathrm{~V}$ for $30 \mathrm{~min}$, and then allowed to cool slowly to room temperature with the electric field applied through continuously during the entire heating and cooling process.

\subsection{The $\{3-3\}$ mode piezoelectric MEMS generator}

Figure 7 shows the fabrication process of the $\{3-3\}$ mode piezoelectric MEMS generator. First, a $5 \mu \mathrm{m}$ PZT layer was deposited onto the SOI wafer using the aerosol deposition method and then patterned and annealed identically to the fabrication process of the $\{3-1\}$ mode device. After the PZT layer was deposited, a $30 \mathrm{~nm}$ Ti and $220 \mathrm{~nm}$ Pt layer were deposited onto the top of the wafer using an e-beam evaporator. The interdigitated electrode was patterned by a lift-off process. The beam shape was defined by a top-side DRIE etching, and the buried oxide layer was etched out using RIE at the same time. Then, DRIE was used to etch from the back side until the beam was released. The proof mass was made and its size was adjusted during the back side etching step. After the back-side etching process, the beam thickness was about $11 \mu \mathrm{m}$. The device was then poled under $180 \mathrm{~V}$ at $160{ }^{\circ} \mathrm{C}$ for $30 \mathrm{~min}$, and then slowly cooled to room temperature. The (a)

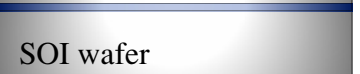

(b)

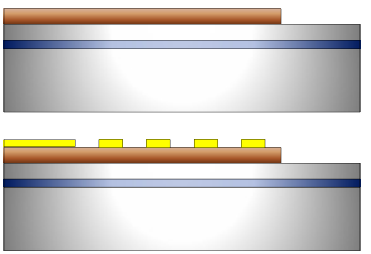

(d)

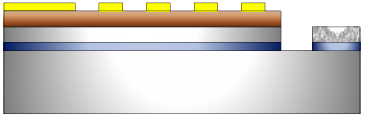

(e)

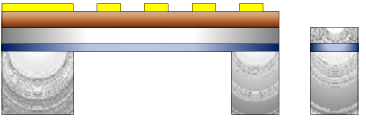

$\mathrm{Si} \square \mathrm{SiO}_{2} \square \mathrm{Ti} / \mathrm{Pt} \square \mathrm{PZT}$

Figure 7. Processing procedure flowchart for $\{3-3\}$ mode piezoelectric MEMS generator: (a) SOI wafer preparation, (b) PZT deposition and patterning, $(c)$ interdigitated electrode deposition and patterning, $(d)$ beam shape etching and $(e)$ back-side etching and released beam.

electric field was applied continuously through the entire heating and cooling process. The SEM of the finished $\{3-1\}$ mode and $\{3-3\}$ mode piezoelectric MEMS generators are shown in figure 8 . The cantilever beam was covered with laminated or interdigitated electrodes and the proof mass at its tip can be seen. The beam structure could be seen to be bent upwards due to the thermal expansion difference for PZT and to the silicon wafer after the PZT cooled down to room temperature from $650{ }^{\circ} \mathrm{C}$.

\section{Experimental set-up and results}

Figure 9 shows the experiment set-up. The piezoelectric MEMS generators were mounted on a shaker (B\&K Type 4809) which acted as the vibration source. The shaker was driven by a function generator in a sinusoidal waveform. The output signal of the piezoelectric MEMS generator was connected to a different resistive load to obtain the relationship between load impedance and output characteristics such as output power and output voltage. A data acquisition device (NI USB-6251) with high input impedance (10 G $\Omega$ ) that was closed to an open circuit condition was used to measure the output characteristics. An accelerometer (B\&K Type 4381) was used to measure the given vibration condition.

When the resonant vibration was achieved, the generator obtained maximum output power under the same load impedance. The output power was highly dependent on the load impedance. Figure 10 shows the relationship between output voltage (peak to peak) and output power at different resistive loads with $1 g$ acceleration for the $\{3-1\}$ mode piezoelectric MEMS generator. The resonant frequency of the $\{3-1\}$ mode piezoelectric MEMS generator was about $255.9 \mathrm{~Hz}$. The maximum peak-to-peak output voltage was 


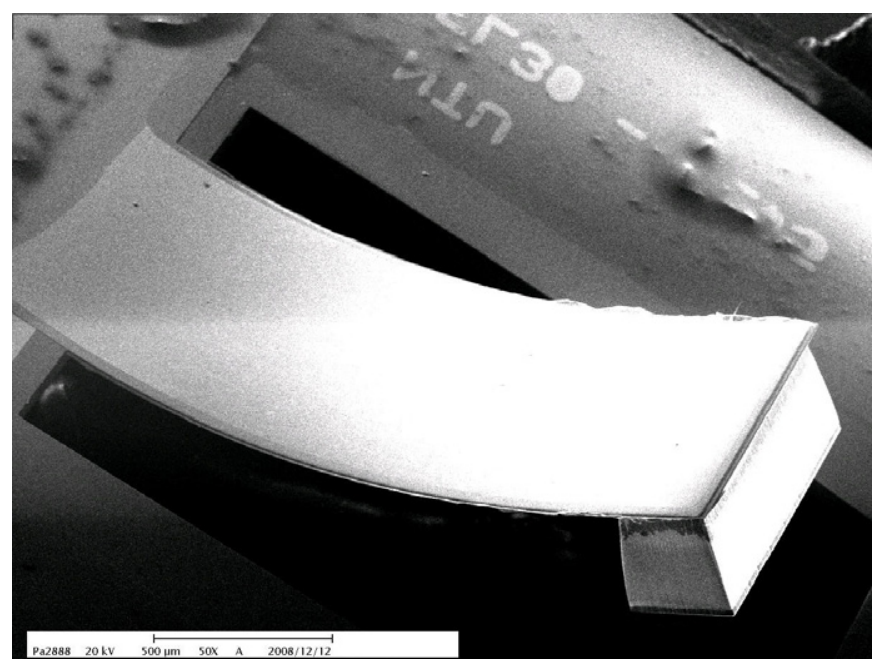

(a)

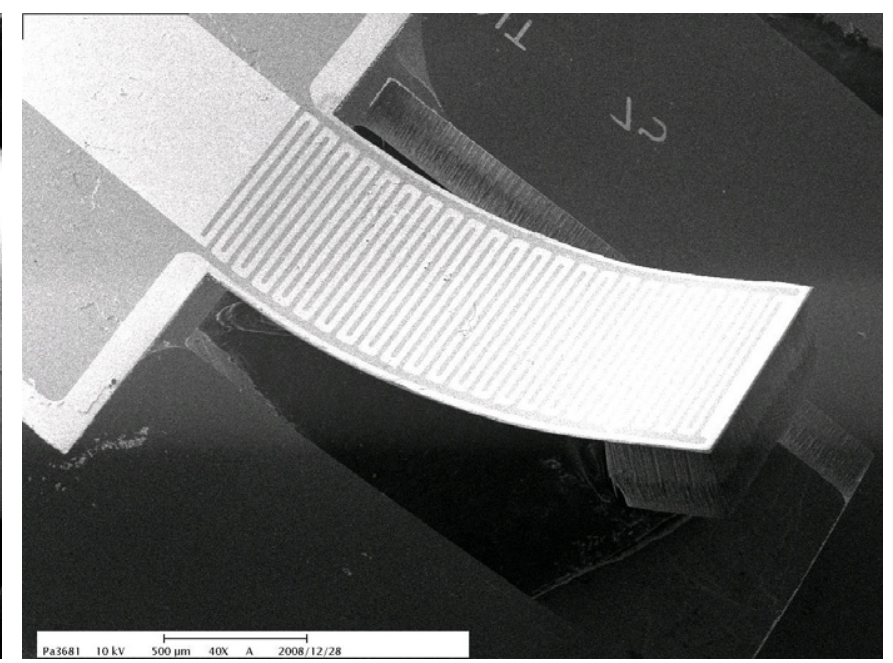

(b)

Figure 8. SEM photograph of the finished piezoelectric MEMS generators: (a) $\{3-1\}$ mode device and $(b)\{3-3\}$ mode device.

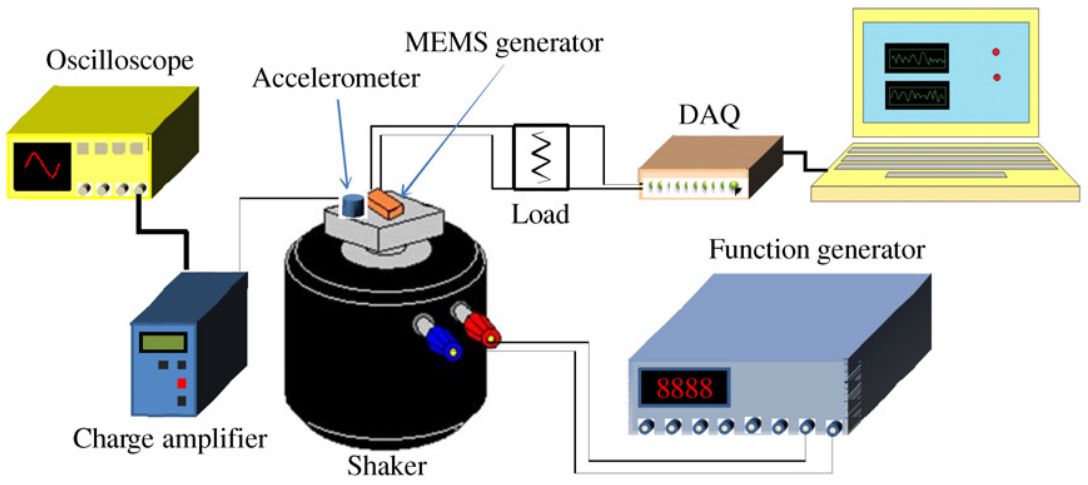

Figure 9. Experimental set-up for the MEMS generators.

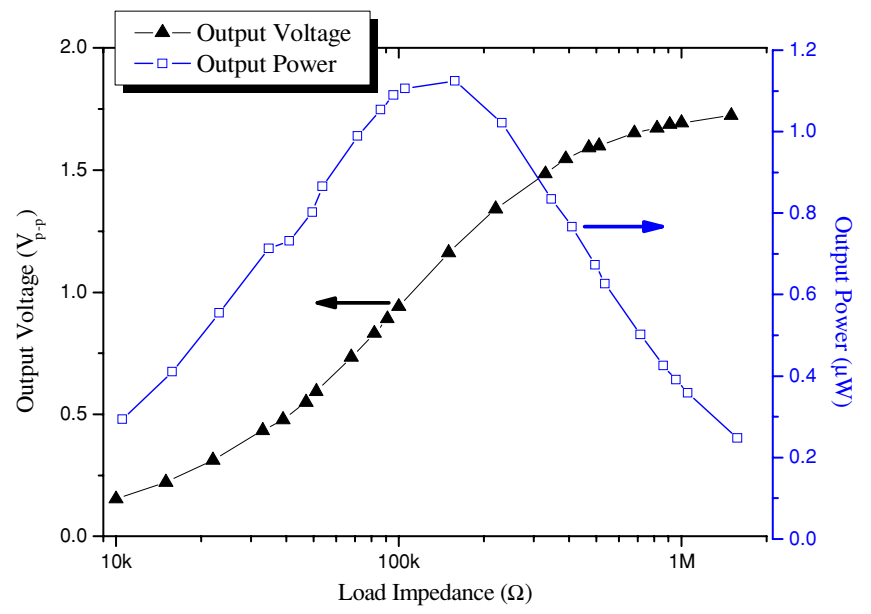

Figure 10. Relationship between output voltage and output power at different resistive loads for $\{3-1\}$ mode piezoelectric MEMS generators excited at $1 g$ acceleration.

$1.723 \mathrm{~V}_{\mathrm{P}-\mathrm{P}}$ at resonant frequency with a $1 \mathrm{~g}$ vibration source under an open circuit condition. The output voltage decreased with respect to a decrease in the resistive load. At a $1 \mathrm{~g}$ vibration, the output power had a peak value of $1.125 \mu \mathrm{W}$ when the load resistance was at $150 \mathrm{k} \Omega$. At this maximum power output condition, the output voltage was $1.162 \mathrm{~V}_{\mathrm{P}-\mathrm{P}}$ that was higher than the minimum requirement for the rectifier circuit diode band gaps. Therefore this opens up the possibility for application as a dc power supply for low power electronic devices.

We found that the maximum output power was dependent on the applied acceleration level. This is to say that the output result of the piezoelectric MEMS generator was a function of the applied acceleration. The $\{3-1\}$ mode piezoelectric MEMS generator was tested up to a $2.5 \mathrm{~g}$ acceleration level. Figure 11 shows the relationship between maximum output power and output voltage at different applied accelerations under a $150 \mathrm{k} \Omega$ resistive load. We found that our device had a maximum output power at $2.675 \mu \mathrm{W}$ with a output voltage of $1.792 \mathrm{~V}_{\mathrm{P}-\mathrm{P}}$ under a matching impedance condition at a $2.5 \mathrm{~g}$ acceleration. Simultaneously, the open circuit output voltage was found to be $2.765 \mathrm{~V}_{\mathrm{P}-\mathrm{P}}$ for this device.

The measured resonant frequency of the $\{3-3\}$ mode piezoelectric MEMS generator was about $214.0 \mathrm{~Hz}$, which was smaller than that of the $\{3-1\}$ mode piezoelectric MEMS generator due to its larger proof mass. Figure 12 shows the relationship between output voltage (peak to peak) and output power at different resistive loads at a $1 g$ acceleration level for the $\{3-3\}$ mode piezoelectric MEMS generator. The maximum peak-to-peak output voltage was $2.392 \mathrm{~V}_{\mathrm{P}-\mathrm{P}}$ at resonant frequency with a $1 \mathrm{~g}$ vibration source under an 
Table 1. Output performance of a piezoelectric MEMS generator at $2 g$ acceleration.

\begin{tabular}{llllll}
\hline Piezoelectric mode & Resonant frequency & Optimal load & Power output & $\begin{array}{l}\text { Voltage output } \\
\text { (open circuit) }\end{array}$ & $\begin{array}{l}\text { Voltage output } \\
\text { (with load) }\end{array}$ \\
\hline$\{3-1\}$ & $255.9 \mathrm{~Hz}$ & $150 \mathrm{k} \Omega$ & $2.099 \mu \mathrm{W}$ & $2.415 \mathrm{~V}$ & $1.587 \mathrm{~V}$ \\
$\{3-3\}$ & $214.0 \mathrm{~Hz}$ & $510 \mathrm{k} \Omega$ & $1.288 \mu \mathrm{W}$ & $4.127 \mathrm{~V}$ & $2.292 \mathrm{~V}$ \\
\hline
\end{tabular}

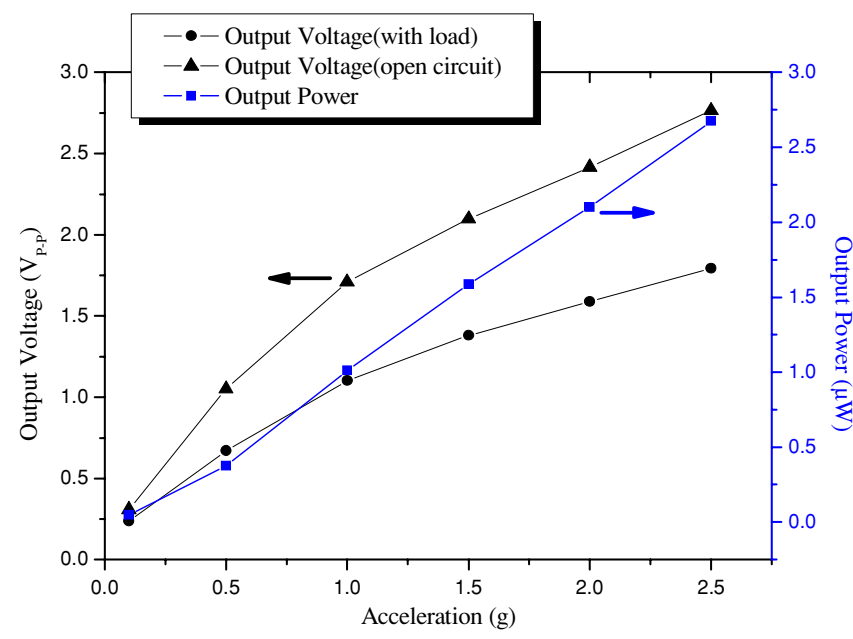

Figure 11. Relationship between maximum output power and output voltage at different applied accelerations levels for $\{3-1\}$ mode piezoelectric MEMS generators with a $150 \mathrm{k} \Omega$ resistive load.

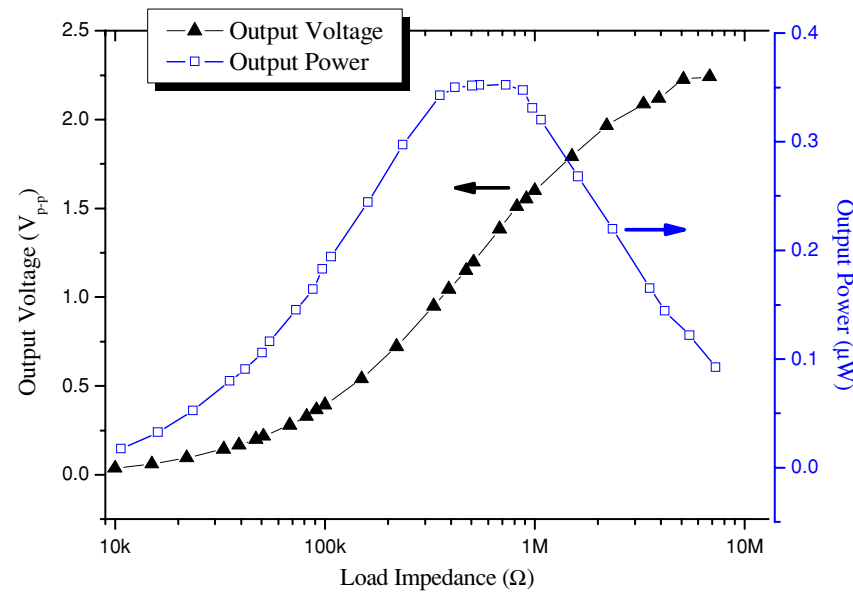

Figure 12. Relationship between output voltage and output power at different resistive loads for $\{3-3\}$ mode piezoelectric MEMS generators excited at $1 g$ acceleration.

open circuit condition. The output voltage was found to decrease with respect to a decrease in the resistive load. At a $1 \mathrm{~g}$ vibration source, the output power had a peak value of $0.352 \mu \mathrm{W}$ when the load resistance was at $510 \mathrm{k} \Omega$. At this maximum power output condition, the output voltage was $1.199 \mathrm{~V}_{\text {P-P }}$ which was also higher than the minimum requirement for the rectifier circuit diode band gaps.

The $\{3-3\}$ mode piezoelectric MEMS generator was tested up to a $2 g$ acceleration level. Figure 13 shows the relationship between maximum output power and output voltage at different applied accelerations under a $510 \mathrm{k} \Omega$ resistive load. We found that our device had a maximum output power of $1.288 \mu \mathrm{W}$ with an output voltage of $2.292 \mathrm{~V}_{\mathrm{P}-\mathrm{P}}$

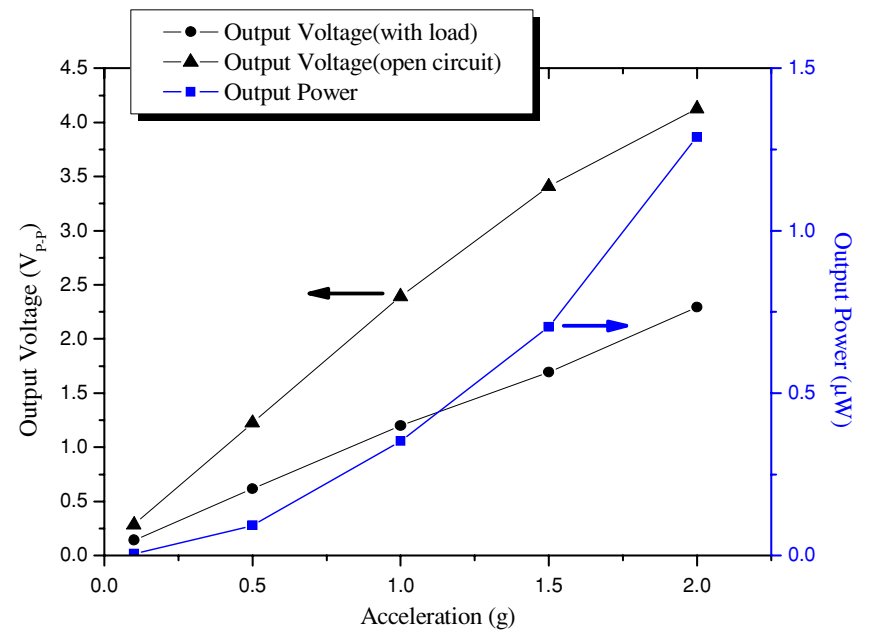

Figure 13. Relationship between maximum output power and output voltage at different applied acceleration levels for $\{3-3\}$ mode piezoelectric MEMS generators with a $510 \mathrm{k} \Omega$ resistive load.

under a matching impedance condition at a $2 g$ acceleration. Simultaneously, the open circuit output voltage was $4.127 \mathrm{~V}_{\mathrm{P}-\mathrm{P}}$ for the $\{3-3\}$ mode piezoelectric MEMS generator.

The experimental results of the $\{3-1\}$ mode and the $\{3-3\}$ mode piezoelectric MEMS generators excited at a $2 g$ acceleration level are summarized in table 1 . As the proof mass of the $\{3-1\}$ mode device was of a size of $500 \times$ $1500 \times 500 \mu \mathrm{m}^{3}$ and the $\{3-3\}$ mode device was of a size of $750 \times 1500 \times 500 \mu \mathrm{m}^{3}$, the resonance frequency of the $\{3-1\}$ mode device was higher than that of the $\{3-3\}$ mode device. The optimal load was thus found to be inversely proportional to the capacitance of the piezoelectric material [18]. For the same dimensions of the beam shape of the $\{3-1\}$ and $\{3-3\}$ mode devices, it was obvious that the capacitance of the $\{3-1\}$ mode device was larger than the $\{3-3\}$ mode device. Therefore, the optimal resistive load for the $\{3-1\}$ mode device was smaller than that of the $\{3-3\}$ mode device. The output power of the $\{3-3\}$ mode piezoelectric MEMS generator was smaller than that of the $\{3-1\}$ mode piezoelectric MEMS generator. This was due to the PZT material of the $\{3-3\}$ mode device which was poled by the interdigitated electrodes and which results in a non-uniform poling direction. The material under the electrodes was not used because it does not pole correctly. Furthermore, the further the distance from the surface of the PZT material, the less effective the poling electric field strength will be. This causes an efficiency drop for the $\{3-3\}$ mode piezoelectric MEMS generator when compared to the \{3-1\} mode piezoelectric MEMS generator. Nevertheless, the output voltage of the $\{3-3\}$ mode piezoelectric MEMS generator was higher than that of the $\{3-1\}$ mode piezoelectric MEMS generator and was easily adjusted by the gap of the 
interdigitated electrodes under the same dimensions of the beam shape.

In order to power a wireless sensor node by using a MEMS power generator, either increasing the total power output or decreasing the power consumption and working duty ratio of a wireless sensor node is a possible way to be realized. To improve the output power of the MEMS generator, an optimal design followed the Roundy's approach can be adopted [8]. On the other hand, Liu et al [19] reported an arrayed MEMS power generator that can increase the total power output by a multi-beam configuration. Decreasing the power consumption of a wireless sensor node is the other way to open the possibility of the MEMS generator to power a wireless sensor node. Marzencki et al [14] reported that a simple wireless sensor node containing a 4 bit RISC microcontroller, a wireless transmitter as well as temperature and acceleration sensors needs only an average power of $150 \mathrm{nW}$ for a low duty cycle operation. This indicated that it generates enough power for a MEMS generator to drive a wireless sensor node.

\section{Conclusions}

In this paper, we presented the design and manufacture methods of two piezoelectric MEMS generators, which have the ability to convert vibration energy into useful electrical energy. These two piezoelectric generators, a $\{3-1\}$ and a $\{3-3\}$ mode piezoelectric MEMS generator, were fabricated using an aerosol deposition method to the PZT layer. For the two piezoelectric mode MEMS generators, we investigated the relationship between output voltage and output power at different resistive loads. We also looked at the relationship between maximum output power and output voltage at different acceleration levels under matching impedance conditions. Our experimental results show that the \{3-1\} mode piezoelectric MEMS generator had a maximum open circuit output voltage of $2.675 \mathrm{~V}_{\mathrm{P}-\mathrm{P}}$ and a maximum output power of $2.765 \mu \mathrm{W}$ with a $1.792 \mathrm{~V}_{\mathrm{P}-\mathrm{P}}$ output voltage at a resonant frequency of $255.9 \mathrm{~Hz}$ at a $2.5 \mathrm{~g}$ acceleration level. The $\{3-3\}$ mode piezoelectric MEMS generator showed a maximum open circuit output voltage of $4.127 \mathrm{~V}_{\text {P-P }}$ and a maximum output power of $1.288 \mu \mathrm{W}$ with a $2.292 \mathrm{~V}_{\text {P-P }}$ output voltage at a resonant frequency of $214 \mathrm{~Hz}$ at a $2 g$ acceleration level. When we compared the output characteristics of both the $\{3-1\}$ mode and the $\{3-3\}$ mode piezoelectric MEMS generators, the results showed that the $\{3-1\}$ mode device, which was made of a PZT sandwiched between laminated electrodes, was better in output power performance than the $\{3-3\}$ mode device which composed of interdigitated electrodes at the top.

\section{Acknowledgment}

The authors would like to acknowledge the financial support for this research from the National Science Council of Taiwan, through Grants NSC 972218E002026, NSC 95-2622-E-002003 and NSC 95-2218-E-002-052.

\section{References}

[1] Elvin N G, Elvin A A and Spector M 2001 A self-powered mechanical strain energy sensor Smart Mater. Struct. 10 293-9

[2] Rabaey J M, Ammer M J, da Silva J L, Patel D and Roundy S 2000 PicoRadio supports ad hoc ultra-low power wireless networking Computer 33 42-8

[3] Roundy S, Wright P K and Rabaey J 2003 A study of low level vibrations as a power source for wireless sensor nodes Comput. Commun. 26 1131-44

[4] Amirtharajah R and Chandrakasan A P 1998 Self-powered signal processing using vibration-based power generation IEEE J. Solid-State Circuits 33 687-95

[5] Roundy S, Steingart D, Frechette L, Wright P and Rabaey J 2004 Power sources for wireless sensor networks Wirel. Sensor Networks, Proc. 2920 1-17

[6] Roundy S, Leland E S, Baker J, Carleton E, Reilly E, Lai E, Otis B, Rabaey J M, Wright P K and Sundararajan V 2005 Improving power output for vibration-based energy scavengers IEEE Pervasive Comput. 4 28-36

[7] Sodano H A 2004 A review of power harvesting from vibration using piezoelectric materials Shock Vib. Dig. 36197

[8] Roundy S and Wright P K 2004 A piezoelectric vibration based generator for wireless electronics Smart Mater. Struct. 13 1131-42

[9] Jeon Y B, Sood R, Jeong J H and Kim S G 2005 MEMS power generator with transverse mode thin film PZT Sensors Actuators A 122 16-22

[10] Williams C B and Yates R B 1996 Analysis of a microelectric generator for microsystems Sensors Actuators A 52 8-11

[11] Fang H B, Liu J Q, Xu Z Y, Dong L, Wang L, Chen D, Cai B C and Liu Y 2006 Fabrication and performance of MEMS-based piezoelectric power generator for vibration energy harvesting Microelectron. J. 37 1280-4

[12] Lee B S, Wu W J, Shih W P, Vasic D and Costa F 2007 Power harvesting using piezoelectric MEMS generator with interdigital electrodes Ultrasonics Symp., 2007. IEEE pp 1598-601

[13] Shen D, Park J H, Ajitsaria J, Choe S Y, Wikle H C and Kim D J 2008 The design, fabrication and evaluation of a MEMS PZT cantilever with an integrated Si proof mass for vibration energy harvesting J. Micromech. Microeng. 18055017

[14] Marzencki M, Ammar Y and Basrour S 2008 Integrated power harvesting system including a MEMS generator and a power management circuit Sensors Actuators A 145 363-70

[15] duToit N E, Wardle B L and Kim S G 2005 Design considerations for MEMS-scale piezoelectric mechanical vibration energy harvesters Integr. Ferroelectr. 71 121-60

[16] Wang X Y, Lee C Y, Peng C J, Chen P Y and Chang P Z 2008 A micrometer scale and low temperature PZT thick film MEMS process utilizing an aerosol deposition method Sensors Actuators A 143 469-74

[17] Wang X Y, Lee C Y, Hu Y C, Shih W P, Lee C C, Huang J T and Chang P Z 2008 The fabrication of silicon-based PZT microstructures using an aerosol deposition method J. Micromech. Microeng. 18055034

[18] Shu Y C and Lien I C 2006 Analysis of power output for piezoelectric energy harvesting systems Smart Mater. Struct. 15 1499-512

[19] Liu J Q, Fang H B, Xu Z Y, Mao X H, Shen X C, Chen D, Liao H and Cai B C 2008 A MEMS-based piezoelectric power generator array for vibration energy harvesting Microelectron. J. 39 802-6 